\title{
HYPERBOLIC MANIFOLDS AND DEGENERATING HANDLE ADDITIONS
}

\author{
MARTIN SCHARLEMANN and YING-QING WU
}

(Received 10 May 1992)

Communicated by J. H. Rubinstein

\begin{abstract}
A 2-handle addition on the boundary of a hyperbolic 3-manifold $M$ is called degenerating if the resulting manifold is not hyperbolic. There are examples that some manifolds admit infinitely many degenerating handle additions. But most of them are not 'basic'. (See Section 1 for definitions). Our first main theorem shows that there are only finitely many basic degenerating handle additions. We also study the case that one of the handle additions produces a reducible manifold, and another produces a $\partial$-reducible manifold, showing that in this case either the two attaching curves are disjoint, or they can be isotoped into a once-punctured torus. A byproduct is a combinatorial proof of a similar known result about degenerating hyperbolic structures by Dehn filling.
\end{abstract}

1991 Mathematics subject classification (Amer. Math. Soc.): primary 57 N 10, secondary 57 M 50 .

\section{Main results and examples}

In this paper, all 3-manifolds are assumed orientable. We work in smooth or PL categories. If $\alpha$ is a subset of a 3-manifold $M$ (generally, a properly embedded submanifold), denote by $N(\alpha)$ a closed regular neighbourhood of $\alpha$, and by $\eta(\alpha)$ an open regular neighbourhood, that is $\eta(\alpha)=N(\alpha)-\overline{M-N(\alpha)}$. If $A$ is a subset of $M$, we use $|A|$ to denote the number of components in $A$. All the

(C) 1993 Australian Mathematical Society 0263-6115/93 \$A2.00+0.00

The first author was partially supported by NSF grant DMS 8901065; and the second by NSF grant DMS 9102633 
concepts and notations not defined in the paper are standard; see, for example $[5,6]$.

Given an essential simple closed curve $\alpha$ on the boundary of $M$, we define $M[\alpha]$ to be the manifold obtained from $M$ by attaching a 2-handle to $M$ along $\alpha$, then capping off a possible 2-sphere component of the resulting manifold by a 3-ball. More explicitly, if $\alpha$ is an essential curve on a torus component of $\partial M, M[\alpha]$ is the Dehn filling along the slope $\alpha$, while if $\alpha$ is on a non-toral component, $M[\alpha]$ is obtained from $M$ simply by attaching a 2-handle along $\alpha$.

Denote by $D M$ the double of $M$ along non-toral, non-spherical boundary components. $M$ is called hyperbolic if the interior of $D M$ admits a complete hyperbolic structure of finite volume. By Thurston's Geometrization Theorem, if $M$ is Haken, (in particular, if $M$ has some non-sphere boundary components), then $M$ is hyperbolic if and only if $M$ is irreducible, $\partial$-irreducible, atoroidal and anannular.

DEFINITION. If $M$ is hyperbolic but $M[\alpha]$ is not, then the handle addition or Dehn filling along $\alpha$ is called a degenerating handle addition or Dehn filling. The curve $\alpha$ on $\partial M$ is called a degenerating curve.

Suppose $T$ is a torus component of $\partial M$, and suppose $M$ is hyperbolic. By a theorem of Thurston, there are only finitely many Dehn fillings on $T$ which yield nonhyperbolic manifolds. In our language, there are only finitely many degenerating curves on $T$. If there is more than one boundary component, one can do Dehn fillings step by step. Since at each step there are only finitely many ways to degenerate the hyperbolic structure, one can say that most Dehn fillings along torus components of $\partial M$ yield hyperbolic manifolds.

Now suppose $F$ is a boundary component of $M$ with genus $g>1$. It is natural to ask whether there are only finitely many handle additions along curves on $F$ that yield non-hyperbolic manifolds. The answer is 'no' in general. Actually, the following is an example that infinitely many handle additions on the boundary of a hyperbolic manifold may yield a solid torus, and the example is easily modified so that infinitely many handle additions yield handlebody of genus $g>1$. We need the following definition and lemma.

DEFINITION. Two curves $\alpha$ and $\beta$ on $F$ are called coplanar if some component of $F-\alpha \cup \beta$ is an annulus or once-punctured annulus.

LEMMA 1.1. Suppose $\alpha$ is a non-separating simple closed curve on $F$. If a separating curve $\beta$ is coplanar to $\alpha$, then $M[\alpha]=M[\beta][\alpha]$. 
In other words, to obtain $M[\alpha]$, we can first attach a 2-handle along the curve $\beta$, which produces a torus boundary component containing $\alpha$, then do Dehn filling along $\alpha$. The lemma is geometrically obvious. We omit the proof.

EXAMPLE 1.1. Consider the manifold $T \times I$, where $T$ is a torus. By a theorem of Myers [7], there exists an arc $\gamma$ in it with one endpoint on each of the boundary components, such that the manifold $M=(T \times I)-\eta(\gamma)$ is hyperbolic. Let $m$ be a curve on $\partial M$ which bounds a disk in $N(\gamma)$. Suppose $\alpha$ is a nonseparating curve on $\partial M$ which is disjoint from $m$. Then, by the lemma, we have $M[\alpha]=M[m][\alpha]$. But since $M[m]=T \times I$, all Dehn fillings produce solid tori. Therefore, $M[\alpha]$ are solid tori for all such $\alpha$. One can also use the trivial link instead of the Hopf link. But then many of the handle additions as above produce connected sums of a solid torus with lens spaces. Figure 1 is a concrete example. The arc $\gamma$ above corresponds to the arc connecting the two components of the link in the picture. The manifolds $M$ is the exterior of the graph, and $m$ is the meridian of the arc. It can be shown that $M$ is hyperbolic.

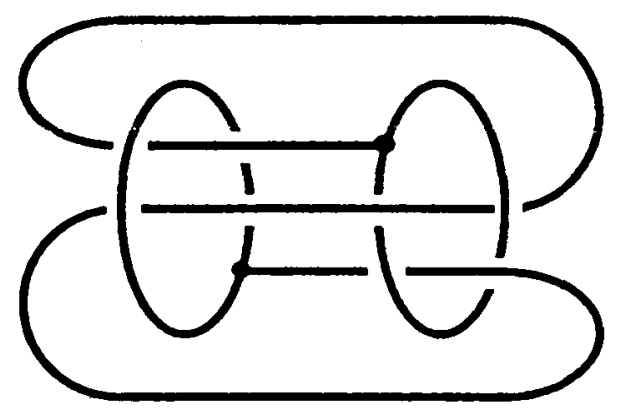

FIGURE 1

Actually, what the example shows is a crucial fact: If $\beta$ is an essential curve on $\partial M$ bounding a punctured torus $P$, such that $M[\beta]$ is non-hyperbolic, then, except in very special cases, most of the Dehn fillings along a curve $\alpha$ in $P$ are non-hyperbolic, so most curves on $P$ are degenerating curves. This leads us to the following.

DEFINITION. A degenerating curve $\alpha$ is called basic if either $\alpha$ is separating, or there are no separating degenerating curves coplanar with $\alpha$. 
Clearly, basic degenerating curves are of primary importance in the study of degenerating handle additions, because any degenerating curve must be coplanar to a basic one. Our first main theorem is the following.

THEOREM 3.4. Suppose $M$ is a hyperbolic 3-manifold. Let $\mathcal{S}$ be the set of basic degenerating curves on a genus $g>1$ boundary component $F$ of $M$. Then $|\mathcal{S}| \leq k_{g}$, where $k_{g}$ is a constant depending only on $g$.

In particular, there are only finitely many separating degenerating curves on $\partial M$. This theorem has immediate application to the following 'handlebody filling' problem. Let $F$ be a genus $g$ boundary component of $M$. Let $H$ be a handlebody of genus $g$. We can glue $H$ to $M$ through a homeomorphism $\varphi: \partial H \rightarrow \partial M$, and call this process a handlebody filling. Let $D_{1}, \ldots, D_{g_{-1}}$ be disks cutting $H$ into $g$ solid tori. Then a handlebody filling can be obtained by first attaching regular neighbourhoods of the $D_{i}$, then attaching the solid tori, so it is decomposed into $g-1$ steps of 2-handle additions along separating curves, followed by $g$ Dehn fillings. At each step there are infinitely many possible choices for the attaching curves, but by the above result and Thurston's Theorem [9, Theorem 5.8.2], only finitely many of them degenerate the hyperbolic structure. Hence we can say that most of the handlebody fillings along $\partial M$ yield hyperbolic manifolds.

On our way towards the proof of Theorem 3.4 , we present a purely combinatorial proof of the following result, which also follows (with better bounds) from the Gromov-Thurston $2 \pi$ theorem cf. [1, Theorem 9]. Suppose $\mathcal{T}=T_{1} \cup \ldots \cup T_{n}$ is a set of tori on the boundary of a hyperbolic 3-manifold $M$. Let $\alpha_{i}$ be a simple closed curve on $T_{i}$. Write $\alpha=\left(\alpha_{1}, \ldots, \alpha_{n}\right)$. Attaching a solid torus on each $T_{i}$ along $\alpha_{i}$ we get a Dehn filled manifold $M[\alpha]=M\left[\alpha_{1}\right] \ldots\left[\alpha_{n}\right]$. It is called a degenerating Dehn filling if $M[\alpha]$ is not hyperbolic. In this case we say that $\alpha$ is a degenerating set. It is called a basic degenerating set if no proper subset of it is degenerating.

PROPOSITION 2.4. Suppose $M$ is hyperbolic manifold, and $\mathcal{T}$ is a set of $n$ tori on $\partial M$. If $\partial M-\mathcal{T}$ is nonempty, then $M$ has at most $C_{n}$ basic degenerating sets, where $C_{n}$ is a constant depending only on $n$.

Next we make deeper investigation of degenerating handle additions. Given two curves $\alpha$ and $\beta$, we use $\Delta(\alpha, \beta)$ to denote their geometric intersection number, that is, the minimal intersection number of $\alpha$ with all $\beta^{\prime}$ which are isotopic to $\beta$. Suppose $\alpha$ and $\beta$ are degenerating curves: what can we say 
about $\alpha$ and $\beta$ ? As we have seen in the example, $\Delta(\alpha, \beta)$ can be arbitrarily large. However, in that example either $\Delta(\alpha, \beta)=0$ or $\alpha$ and $\beta$ lie in a common once-punctured torus. We suspect that this is always true.

In Section 3, we will consider the case that one of the handle additions yields a reducible manifold, and the other one yields a $\partial$-reducible manifold. Consider the following.

EXAMPLE 1.2. Let $K$ be a cable knot in a $\partial$-reducible 3-manifold $X$, such that $X-\eta(K)$ is irreducible and $\partial$-reducible. Let $\alpha$ be a meridian of $K$, and let $\beta$ be the slope of the cabling annulus. Choose an $\operatorname{arc} \gamma$ from $\partial X$ to the torus $T=\partial N(K)$, complicated enough so that $M=(X-\eta(K))-\eta(\gamma)$ is hyperbolic. Then we have that $M[\alpha]=X$ is $\partial$-reducible, and $M[\beta]=(X-\eta(K))[\beta]$ is reducible, but $\Delta(\alpha, \beta) \neq 0$. However, in this case both $\alpha, \beta$ lie on a common punctured torus $T-\eta(\gamma)$.

Our second main theorem shows that this is always the case.

THEOREM 4.2. Suppose $M$ is a hyperbolic manifold, $\alpha, \beta$ are essential simple closed curves on a non-torus boundary component of $M$, such that $M[\alpha]$ is reducible and $M[\beta]$ is $\partial$-reducible. Then either $\Delta(\alpha, \beta)=0$ or both $\alpha$ and $\beta$ can be isotoped into a once-punctured torus $P$ on $\partial M$.

As a corollary, we will see that if either $\alpha$ or $\beta$ is a basic degenerating curve, in particular, if one of them is separating on the surface, then $\Delta(\alpha, \beta)=0$. It will be shown that essentially Example 1.2 has given all the possible manifolds for the second possibility in the theorem.

\section{Basic degenerating Dehn Fillings}

Let $P, Q$ be two properly embedded surfaces in a 3-manifold $M$. We say that they are in minimal intersection position if

(1) $|\partial P \cap \partial Q| \leq\left|\partial P \cap \partial Q^{\prime}\right| \quad$ for all $Q^{\prime}$ isotopic to $Q$, and

(2) $|P \cap Q| \leq\left|P \cap Q^{\prime}\right| \quad$ for all $Q^{\prime}$ subject to (1)

Let $\hat{P}$ be $P$ with each boundary component identified to a point. The image of $P \cap Q$ in $\hat{P}$ can be considered as a graph, denoted by $\Gamma_{P}:$ an edge of the graph is an arc component of $P \cap Q$, and a vertex is the image of a component of $\partial P$ Similarly, we have a graph $\Gamma_{Q}$ in $\hat{Q}$. The edges $e_{1}, e_{2}$ are parallel in $\Gamma_{P}$ if there is a disk $D$ in $\hat{P}$ such that $\partial D=e_{1} \cup e_{2}$, and Int $D$ contains no vertices of $\Gamma_{P}$. 
An edge of $\Gamma_{P}$ is called a trivial loop if it contains only one vertex, and bounds a disk in $\hat{P}$ with interior disjoint from $\Gamma_{P}$. Recall that a properly embedded surface in $M$ is called essential if it is incompressible and $\partial$-incompressible.

LemMa 2.1. Let $M$ be an irreducible, d-irreducible 3-manifold. Suppose $P, Q$ are essential surfaces properly embedded in $M$, isotoped so that they are in minimal intersection position. If there are arcs $e_{1}, e_{2}$ in $P \cap Q$ which are parallel in both $\Gamma_{P}$ and $\Gamma_{Q}$, then $M$ contains an essential annulus.

PROOF. The arcs $e_{1}, e_{2}$ cut off a disk $D_{1}$ from $P$, and a disk $D_{2}$ from $Q$. By re-choosing the edges $e_{i}$ if necessary, we may assume that $D_{1}$ and $D_{2}$. have disjoint interiors. So $A=D_{1} \cup D_{2}$ is a properly embedded annulus or Mobius band in $M$.

If $A$ is a Mobius band, let $N(A)$ be a regular neighbourhood of $A$. The frontier of $N(A)$ is a properly embedded annulus $B=N(A) \cap E(A)$, where $E(A)=M-\eta(A)$. Notice that $N(A)$ is a solid torus, with $B$ running twice along the longitude direction, so $B$ is incompressible and $\partial$-incompressible in $N(A)$. If $B$ is compressible in $E(A)$ with $D$ a compressing disk, then the union of $N(A)$ and a regular neighbourhood of $D$ will be a punctured lens space, which is impossible because $M$ is irreducible and has nonempty boundary. If $B$ is $\partial$-compressible, then a $\partial$-compressing of $B$ would produce a disk, cutting $M$ into two pieces, one of which is a solid torus. Therefore $M$ is $\partial$-compressible, again a contradiction.

Now suppose $A$ is an annulus. Let $c$ be a boundary component of $A$. One can see that $c$ is the union of two $\operatorname{arcs} c_{1}$ and $c_{2}$, where $c_{1} \subset \partial P$, and $c_{2} \subset \partial Q$. If $A$ is compressible, then $c$ bounds a disk in $M$. Since $M$ is $\partial$-irreducible, $c$ bounds a disk on $\partial M$, which can be used to reduce $|\partial P \cap \partial Q|$ by isotoping $c_{1}$ off $c_{2}$. If $A$ is $\partial$-reducible, one can choose a $\partial$-reducing disk $D$ so that the $\operatorname{arc} D \cap A$ lies on $D_{1}$ and is parallel to the edges $e_{1}$, so it is an essential arc on $P$. The disk $D$ may have other intersection with $P$, but by an innermost circle-outermost arc argument one can show that $P$ is either reducible or $\partial$-reducible, which contradicts the hypothesis of $P$. Therefore $A$ is an essential annulus.

LEMMA 2.2. Suppose $P$ and $Q$ are essential punctured spheres or punctured tori in an irreducible, $\partial$-irreducible manifold $M$. Suppose $\partial P$ has $c_{1}$ components parallel to a curve $\gamma_{1}$, and $\partial Q$ has $c_{2}$ components parallel to a curve $\gamma_{2}$. If $\Delta\left(\gamma_{1}, \gamma_{2}\right)>18|\partial P \| \partial Q| / c_{1} c_{2}$, than $M$ contains an essential annulus. 
Proof. By an isotopy, we may assume that $P$ and $Q$ are in minimal intersection position. As before, use $\Gamma_{P}$ to denote the corresponding graph of $P \cap Q$ in $\hat{P}$. If $\Gamma_{P}$ has a trivial loop, then some arc of $P \cap Q$ would be boundary parallel in $P$, so it cuts off a disk from $P$ which can be used to $\partial$-compress $Q$. But since $Q$ is $\partial$-incompressible and $M$ is $\partial$-irreducible, one can find an isotopy of $Q$ reducing $|\partial P \cap \partial Q|$, which is impossible because $P$ and $Q$ are assumed in minimal intersection position. Hence $\Gamma_{P}$ and $\Gamma_{Q}$ have no trivial loops. Similarly, one can show that $P \cap Q$ has no trivial circle (that is, a circle which bounds a disk in $P$ or $Q$ ).

Consider the intersection of $\partial P$ and $\partial Q$ on $T_{1}$. Let $\Delta=\Delta\left(\gamma_{1}, \gamma_{2}\right)$. Since $\partial P$ has $c_{1}$ components parallel to $\gamma_{1}$, and $\partial Q$ has $c_{2}$ components parallel to $\gamma_{2}$, these components intersect at $c_{1} c_{2} \Delta$ points, so $|\partial P \cap \partial Q| \geq c_{1} c_{2} \Delta$. It follows that $\Gamma_{P}$ contains at least $c_{1} c_{2} \Delta / 2$ edges.

Denote by $\tilde{\Gamma}_{P}$ the reduced graph of $\Gamma_{P}$, which by definition is obtained from $\Gamma_{P}$ by replacing a set of parallel edges by a single edge. Then $\tilde{\Gamma}_{P}$ is a graph in $\hat{P}$ with no trivial loops or parallel edges. Denote by $v, e, f$ the number of vertices, edges, and faces of $\tilde{\Gamma}_{P}$, respectively. (A face of $\tilde{\Gamma}_{P}$ is a component of $\hat{P}-\tilde{\Gamma}_{P}$.) The above shows that each face is incident to a least three edges, so we have $3 f \leq 2 e$. Since $\hat{P}$ is either a sphere or a torus, by counting the Euler characteristic we have

$$
v-e+f \geq 0 .
$$

Using the fact that $v=v(P)=|\partial P|$ and $3 f \leq 2 e$, we get

$$
|\partial P|-e+(2 / 3) e \geq 0,
$$

or equivalently, $e \leq 3|\partial P|$. Since $\Gamma_{P}$ has at least $c_{1} c_{2} \Delta / 2$ edges, we see that some edge of $\tilde{\Gamma}_{P}$ corresponds to at least $c_{1} c_{2} \Delta / 6|\partial P|$ parallel edges.

Let $e_{1}, \ldots e_{k}$ be a set of parallel edges in $\Gamma_{P}$. Consider the subgraph $\Gamma_{Q}^{\prime}$ of $\Gamma_{Q}$ with these $e_{i}$ as edges. By the same argument as above, one can show that if $k>3|\partial Q|$ then $\Gamma_{Q}^{\prime}$ has some parallel edges. If $M$ contains no essential annulus, then by Lemma 2.1 no pair of edges could be parallel in both $\Gamma_{P}$ and $\Gamma_{Q}$. Hence $k \leq 3|\partial Q|$. Since $\Gamma_{P}$ has a set of at least $c_{1} c_{2} \triangle / 6|\partial P|$ parallel edges, this gives $c_{1} c_{2} \Delta / 6|\partial P| \leq 3|\partial Q|$, or equivalently, $\triangle \leq 18|\partial P \| \partial Q| / c_{1} c_{2}$.

LEMMA 2.3. Let $\mathcal{S}$ be a set of mutually non-isotopic simple closed curves on a torus $T$. If $\Delta(\alpha, \beta) \leq k$ for all $\alpha, \beta$ in $\mathcal{S}$, then $|\mathcal{S}| \leq 2(k+1)^{2}$.

ProOf. Let $m, l$ be a meridian-longitude pair in $T$. They form a basis for $H_{1}(T)$. We write $\alpha=(a, b)$ if $\alpha=a m+b l$ in $H_{1}(T)$, and by reversing the 
orientation we may always assume $a \geq 0$. If $\alpha=(a, b)$ and $\beta=(c, d)$, their intersection number is given by

$$
\Delta(\alpha, \beta)=\left|\operatorname{det}\left(\begin{array}{ll}
a & b \\
c & d
\end{array}\right)\right|=|a d-b c| .
$$

Fix two elements $\alpha, \beta \in \mathcal{S}$. Without loss of generality, we may assume $\alpha=(0,1)$ and $\beta=(a, b)$, where $a \geq 1$. Now if $\gamma=(c, d) \in \mathcal{S}$, then $\Delta(\alpha, \gamma)=|c| \leq k$ implies $0 \leq c \leq k$, and $\Delta(\beta, \gamma) \leq k$ implies $-k \leq a d-b c \leq k$, or $-k+b c \leq d \leq k+b c$. So $c$ has at most $k+1$ choices and $d$ has fewer than $2(k+1)$ choices. Therefore $|\mathcal{S}| \leq 2(k+1)^{2}$.

Suppose $M$ is a hyperbolic 3-manifold. Let $\mathcal{T}=T_{1} \cup \ldots \cup T_{n}$ be a union of tori on $\partial M$. Suppose $\alpha=\left(\alpha_{1}, \ldots, \alpha_{n}\right)$, where $\alpha_{i}$ is an essential curve on $T_{i}$. Recall from Section 1 that we use $M[\alpha]$ to denote the manifold obtained from $M$ by Dehn fillings along $\alpha$, that is, $M[\alpha]=M\left[\alpha_{1}\right] \ldots\left[\alpha_{n}\right]$. The set $\alpha$ is called a degenerating set if $M[\alpha]$ is non-hyperbolic, and it is a basic degenerating set if no proper subset of $\alpha$ is degenerating. The corresponding Dehn filling will be called degenerating or basic degenerating accordingly.

Proposition 2.4. Suppose $M$ is a hyperbolic manifold. If $\partial M$ has other components than $\mathcal{T}$, then $M$ has at most $C_{n}$ basic degenerating Dehn fillings, where $C_{n}$ is a constant depending only on $n$.

PROOF. We prove the theorem by induction. When $n=1$, by Gordon's Theorem [2], [3, Theorem 3.4] if both $\alpha_{1}$ and $\beta_{1}$ are degenerating curves on $T-1$ then $\Delta\left(\alpha_{1}, \beta_{1}\right) \leq 8$. By Lemma 2.3, we may take $C_{1}=162$. So we assume $n \geq 2$, and suppose $C_{n-1}$ has been defined to satisfy the theorem. Write

$$
\mathcal{S}=\{\alpha \mid \alpha \text { is a degenerating set }\}
$$

Let $\mathcal{S}^{\prime}$ be a maximal subset of $\mathcal{S}$ such that if $\left(\alpha_{1}, \ldots, \alpha_{n}\right)$ and $\left(\beta_{1} \ldots, \beta_{n}\right)$ are both in $\mathcal{S}^{\prime}$ then $\alpha_{i} \neq \beta_{i}$ for all $i$.

LEMMA 2.5. $|\mathcal{S}| \leq n C_{n-1}\left|\mathcal{S}^{\prime}\right|$

PROOF. For each $\alpha \in \mathcal{S}^{\prime}$, let $\mathcal{S}\left(\alpha_{i}\right)=\left\{\beta \in \mathcal{S} \mid \beta_{i}=\alpha_{i}\right\}$. Clearly, $\mathcal{S}=$ $\bigcup\left\{\mathcal{S}\left(\alpha_{i}\right) \mid \alpha \in \mathcal{S}^{\prime}, i=1, \ldots, n\right\}$ so we need only show that $\left|\mathcal{S}\left(\alpha_{i}\right)\right| \leq C_{n-1}$. Without loss of generality, we may assume $i=1$. Then for any $\beta=$ 
$\left(\alpha_{1}, \beta_{2}, \ldots, \beta_{n}\right) \in S\left(\alpha_{i}\right)$, we have $M[\beta]=M\left[\alpha_{1}\right]\left[\beta^{\prime}\right]$, where $\beta^{\prime}=\left(\beta_{2} \ldots, \beta_{n}\right)$. Since $\alpha$ is basic, $M\left[\alpha_{1}\right]$ is hyperbolic, so by definition of $C_{n-1}$ the set $\left\{\beta^{\prime} \mid \beta \in\right.$ $\left.\mathcal{S}\left(\alpha_{1}\right)\right\}$ has at most $C_{n-1}$ elements.

Since $M[\alpha]$ is a degenerating Dehn filling, and $\partial M-\mathcal{T}$ is nonempty, $M[\alpha]$ is either reducible, $\partial$-reducible, toroidal or annular. In all cases, there is a properly embedded surface $F$ in $M[\alpha]$, which is either a reducing sphere, a $\partial$-reducing disk, an essential annulus or an essential torus. We call $F$ a degenerating surface. Let $P=F \cap M$. It is a punctured torus or a punctured sphere. When $F$ is a sphere or torus, $\partial P$ lies on $\mathcal{T}$; when $F$ is a disk or annulus, $\partial P$ has at most two components lying on $\partial M-\mathcal{T}$.

LEMMA 2.6. $F$ can be chosen so that $P=F \cap M$ is an essential surface.

Proof. Among all degenerating surfaces, choose $F$ so that $|F \cap \mathcal{T}|$ is minimal. If $P$ is compressible, let $D$ be a compressing disk. Since $F$ is incompressible, $\partial D$ is inessential in $F$, so a 2-surgery of $F$ along $D$ yields two surfaces, one of which must be a degenerating surface with fewer intersections with $\mathcal{T}$, contradicting the choice of $F$.

Suppose $P$ is $\partial$-compressible. Let $D$ be a $\partial$-compressing disk. $\partial D$ consists of two arcs $e_{1}$ and $e_{2}$, with $e_{1}$ on $P$ and $e_{2}$ on $\partial M$. If $e_{2}$ is on $\mathcal{T}$, part of $F$ can be isotoped through $D$ and the attached solid torus to reduce $|F \cap \mathcal{T}|$. If $e_{2}$ is on $\partial M-\mathcal{T}$, then $F$ must be either a compressing disc or an essential annulus, so $e_{2}$ is an inessential arc in $F$. Hence 2-surgery of $F$ along $D$ yields two surfaces, one of which is a degenerating surface with fewer intersections with $\mathcal{T}$.

Fix a degenerating surface $F_{\alpha}$ for each $\alpha$, so that $P_{\alpha}=F_{\alpha} \cap M$ is incompressible and $\partial$-incompressible. Let

$$
\mathcal{S}_{i}=\left\{\alpha:\left|\partial P_{\alpha} \cap T_{i}\right| \geq\left|\partial P_{\alpha} \cap T_{j}\right| \text { for all } j\right\} .
$$

Then $\mathcal{S}^{\prime}=\bigcup_{i=1}^{n} \mathcal{S}_{i}$. We need to show that each $\mathcal{S}_{i}$ is a finite set.

Suppose $\alpha, \beta$ are two elements in $\mathcal{S}_{i}$. By definition, $P_{\alpha} \cap T_{i}$ has no fewer components than $P_{\alpha} \cap T_{j}$ for all $j$, so $\left|P_{\alpha} \cap \mathcal{T}\right| \leq n\left|P_{\alpha} \cap T_{i}\right|$. Also, $\partial P$ has at most two components not on $\mathcal{T}$. Therefore,

$$
|\partial P| \leq 2+n\left|\partial P \cap T_{i}\right| \leq 2 n\left|\partial P \cap T_{i}\right| .
$$

A similar formula holds for $Q$. Since $M$ is hyperbolic, by Lemma 2.2 we have

$$
\Delta\left(\alpha_{i}, \beta_{i}\right) \leq 18|\partial P||\partial Q| /\left|\partial P \cap T_{i}\right|\left|\partial Q \cap T_{i}\right| \leq 72 n^{2} .
$$


Recall that if $\alpha, \beta$ are different elements in $\mathcal{S}_{i}$, then $\alpha_{i}$ and $\beta_{i}$ are different, so the set $\left\{\alpha_{i} \mid \alpha \in \mathcal{S}_{i}\right\}$ has the same cardinality as that of $\mathcal{S}_{i}$. Therefore, by Lemma 2.3 we have $\left|\mathcal{S}_{i}\right| \leq 2\left(72 n^{2}+1\right)^{2}$.

Combining with Lemma 2.5 , this gives

$$
|\mathcal{S}| \leq n C_{n-1}\left|\mathcal{S}^{\prime}\right| \leq n C_{n-1}\left(\left|\mathcal{S}_{1}\right|+\ldots+\left|\mathcal{S}_{n}\right|\right) \leq 2 n^{2}\left(72 n^{2}+1\right)^{2} C_{n-1} .
$$

The right hand side depends only on $n$, the number of components in $\mathcal{T}$. This completes the proof of Proposition 2.4

\section{Basic degenerating handle additions}

In this section we apply the techniques in the previous section to show that a hyperbolic manifold admits only finitely many basic degenerating handle additions. The proof is quite similar to that of Theorem 2.4, but some difference arises in finding the essential surfaces. Actually, it is generally impossible to find an essential degenerating surface with most boundary components parallel to the degenerating curves. This is amended by considering the "major boundary slope' instead (see the proof of Theorem 3.4 for definition).

The following lemma and its corollary show that on a given surface there are only finitely many curves with mutual intersection number bounded above. This is easy for a given set, but it is not so simple to find a universal bound. Note that the constant $C(g, k)$ in Corollary 3.2 is independent of the set $\mathcal{C}$.

DEFINITION. For $S$ a compact orientable surface, and $\Lambda$ a set of points in $\partial S$, a $\Lambda$-curve $\Gamma$ in $S$ is a compact 1 -manifold properly embedded in $S$ so that $\partial \Gamma \subset \Lambda$, every closed component is essential, and no two closed components are parallel.

If $\Gamma$ and $\Gamma^{\prime}$ are two $\Lambda$-curves in $S$, define

$$
\begin{array}{r}
\Delta\left(\Gamma, \Gamma^{\prime}\right)=\max \left\{\Delta\left(\gamma, \gamma^{\prime}\right) \mid \gamma \text { and } \gamma^{\prime}\right. \text { are components of } \\
\left.\Gamma \text { and } \Gamma^{\prime} \text { respectively }\right\} .
\end{array}
$$

LEMMA 3.1. Given a compact orientable surface $S$, a set $\Lambda$ of points in $\partial S$, and $k>0$, there is an integer $L(S, \Lambda, k)$ such that, if $\left\{\Gamma_{i} \mid 1 \leq i \leq n\right\}$ is a set of mutually non-isotopic $\Lambda$-curves in $S$ so that each $\Delta\left(\Gamma_{i}, \Gamma_{j}\right) \leq k$, then $n \leq L(S, \Lambda, k)$. 
PROOF. If suffices to prove the lemma for $S$ connected. The proof is by induction on the complexity of $S$, as measured, for example, by $3 \operatorname{genus}(S)+|\partial S|$.

If $S$ is a disk $D$, then $k$ is irrelevant, for $\Delta\left(\Gamma_{i}, \Gamma_{j}\right) \leq 1$. Moreover, since a pair of points in $\Lambda$ bounds a unique arc in $D$, the set of possible isotopy classes for $\Lambda$-curves is clearly bounded above by the number of ways of dividing up subsets of $\Lambda$ into pairs of points.

If $D$ is altered by removing a small disk in the interior to create an annulus $A$, then each pair of points in $\Lambda$ bounds two arcs in $A$, and $A$ contains a single essential simple closed curve. Thus, again, there is an obvious bound $b(|\Lambda|)$ to the number of possible $\Lambda$-curves in $A$.

More generally, if $S$ is an arbitrary compact connected orientable surface, and each component of each $\Gamma_{i}$ is $\partial$-parallel, then all the $\Gamma_{i}$ lie in an annular neighbourhood of $\partial S$. This implies that $n \leq b(|\Lambda|)^{|\partial S|}$.

Thus the lemma is proven unless some component of some $\Gamma_{i}$ is not $\partial$-parallel in $S$. If there is such a component $\gamma$, let $\kappa$ be a set of $k$ points in $\gamma$. Let $S^{\prime}$ be the surface obtained by removing an open regular neighbourhood $\eta(\gamma)$ of $\gamma$ from $S$. Then $\partial S^{\prime}$ is obtained from $\partial S$ by removing a neighbourhood of $\partial \gamma$ and adding two copies $\gamma_{ \pm}$of $\gamma$. Let $\Lambda^{\prime} \subset \partial S^{\prime}$ be $\Lambda-\partial \gamma$ together with a copy $\kappa_{ \pm}$of $\kappa$ in each of $\gamma_{ \pm}$.

Define $\Gamma_{1}^{\prime} \subset S^{\prime}$ to be $\Gamma_{1}-\gamma$. For each $i>1$, isotope $\Gamma_{i}$ to intersect $\gamma$ minimally and define $\Gamma_{i}^{\prime} \subset S^{\prime}$ to be $\Gamma_{i}-\eta(\gamma)$. Note that each component of $\Gamma_{i}^{\prime}$ lies in a component of $\Gamma_{i}$, so each component of $\Gamma_{i}^{\prime}$ intersects each component of $\Gamma_{j}^{\prime}$ in at most $k$ points. Each $\Gamma_{i}^{\prime}$ has at most $k$ boundary points on each of $\gamma_{ \pm} \subset \partial S^{\prime}$, but of course they do not automatically lie in $\kappa_{ \pm}$. This can be fixed by ambiently isotoping the set of points $\left(\Gamma_{i} \cap \gamma\right), i>1$, into a subset of $\kappa$. Even when $\gamma$ is a circle, this isotopy can be chosen so no point passes more than once through the same point of $\kappa$. In particular, this isotopy introduces at most one new intersection between an end of an arc in $\Gamma_{i}^{\prime}$ and an end of an arc in $\Gamma_{j}^{\prime}$, so $\Delta\left(\Gamma_{i}^{\prime}, \Gamma_{j}^{\prime}\right) \leq k+4$.

Now an isotopy from $\Gamma_{i}^{\prime}$ to $\Gamma_{j}^{\prime}$ in $S^{\prime}$ is the restriction of an isotopy of $\Gamma_{i}$ to $\Gamma_{j}$ relative to their identical intersection with $\gamma$. Hence we know that the $\left\{\Gamma_{j}^{\prime}\right\}$ are mutually non-isotopic in $S^{\prime}$. Each component of $S^{\prime}$ is simpler than $S$, so, by inductive hypothesis, there is a number $L\left(S^{\prime}, \Lambda^{\prime}, k+4\right)$ so that $n \leq$ $L\left(S^{\prime}, \Lambda^{\prime}, k+4\right)$. The proof is then completed by observing that there are only a finite number of surfaces of at most two components, each simpler than $S$, and in each of these there are only a finite number of ways of distributing $|\Lambda|+2 k$ points into the boundary. 
COROLLARY 3.2. There is a constant $C(g, k)$ such that if $\mathcal{C}$ is a set of mutually non-isotopic simple closed curves on a genus $g$ closed orientable surface $F$ with $\triangle(\alpha, \beta) \leq k$ for all $\alpha, \beta, \in \mathcal{C}$, than $|\mathcal{C}| \leq C(g, k)$.

PROOF. Regard each simple closed curve as a $\Lambda$-curve, $\Lambda=\emptyset$, on the genus $g$ surface $\mathcal{S}$, and define $C(g, k)=L(S, \emptyset, k)$.

LEMMA 3.3. Suppose $\alpha$ is a degenerating curve on $F$. Then there is a punctured sphere or torus $P$ in $M$ such that $P$ is essential, and all but possibly two boundary components of $P$ are coplanar with $\alpha$.

Proof. Suppose $P$ is a surface in $M$. If $\partial$ is a component of $\partial P$ and is coplanar with $\alpha$, then $\partial$ bounds a disk in $M[\alpha]$. Capping off all such components by mutually disjoint disks in $M[\alpha]$, we get a surface $\hat{P}$ in $M[\alpha]$. The surface $P$ is called a presurface if $\hat{P}$ is a degenerating surface. We assume $\alpha$ is nonseparating. The proof of the other case is similar and simpler.

Write $\partial P=\hat{\partial} \cup \partial_{1} \cup \ldots \cup \partial_{\mathcal{S}} \cup \partial_{1}^{\prime} \cup \ldots \cup \partial_{t}^{\prime}$ where $\hat{\partial}=\partial \hat{P}$, the $\partial_{i}$ are parallel to $\alpha$, and the $\partial_{j}^{\prime}$ are coplanar but not parallel to $\alpha$. We label the components so that $\partial_{i}$ is adjacent to $\partial_{i+1}, \partial_{j}^{\prime}$ is adjacent to $\partial_{j+1}^{\prime}$, and $\partial_{1}^{\prime}$ is the closest one to the $\partial_{i}$. Define

$$
b(\hat{P})= \begin{cases}1 & \text { if } \hat{P} \text { is a disk; } \\ 2 & \text { if } \hat{P} \text { is an annulus; } \\ 3 & \text { if } \hat{P} \text { is a torus. }\end{cases}
$$

Define the complexity of $P$ to be $c(P)=(b(\hat{P}),|\partial P|)$ in lexicographic order. Since $\alpha$ is degenerating, presurfaces do exist. Let $P$ be one with least complexity. Clearly, $P$ is incompressible, for a compression of $P$ would produce a presurface of lower complexity.

Now suppose $P$ is $\partial$-compressible, with $D$ a $\partial$-compressing disk. Write $\partial D=u \cup v$, where $u$ is an arc in $F$, and $v$ is an arc in $P$. Since $P$ is incompressible, $u$ cannot be relative to $\partial u$ isotoped into $\partial P$. In other words, $(u, \partial u)$ is essential in $(F, \partial P)$. There are several cases.

(1) $u$ has endpoints on different components of $\partial P$;

(2) $t>0, s=1$ and $\partial u \subset \partial_{1}$;

(3) $\partial u \subset \hat{\partial}$;

(4) $\partial u \subset \partial_{1}^{\prime}$;

(5) $\partial u \subset \partial_{t}^{\prime}$, or $t=0, \partial u \subset \partial_{1}$ or $\partial_{s}$.

$\partial$-compressing $P$ along $D$, we get a new surface, which has one or two new 
boundary components, depending on whether the two ends of $u$ lie on different components of $\partial P$. If a new boundary component is trivial in $F$, we cap off the component by a disk: in this way, we get a new surface, denoted by $P^{\prime}$. We will show that Case (2) is impossible, and in all other cases $P^{\prime}$ has a component which is a presurface with lower complexity than $P$.

In Case (1), since $\hat{P}$ is $\partial$-incompressible, $u$ cannot have both ends on $\hat{\partial}$. For all the other possibilities, one can see that $\hat{P}^{\prime}=\hat{P}$ and $\left|\partial P^{\prime}\right|<|\partial P|$.

In Case (2), a regular neighbourhood of $v$ and a disk bounded by $\partial_{1}$ would form a Mobius band in $\hat{P}$, which is absurd.

In Case (3), since $\hat{P}$ is $\partial$-incompressible, $\hat{P}^{\prime}$ must have a component isotopic to $\hat{P}$, so the corresponding component of $P^{\prime}$ is a presurface of lower complexity.

In Case (4), the two new components of $P^{\prime}$ are parallel to $\alpha$. They bound disks in $M[\alpha]$. Since $\hat{P}$ is incompressible, they are inessential in $\hat{P}$, so $v$ is a separating arc. Therefore, $P^{\prime}$ has two components, one of which is the required surface.

In Case (5), the new boundary components are essential curves on $\partial M[\alpha]$, so $\left|\partial \hat{P}^{\prime}\right|=|\partial \hat{P}|+2$. Thus either $\hat{P}^{\prime}$ has a compressing disk component for $\partial M[\alpha]$, in which case the corresponding component of $P^{\prime}$ is a presurface of lower complexity, or $\hat{P}^{\prime}$ is one or two annuli. One can see that $\hat{P}^{\prime}$ can be obtained from $\hat{P}$ by 2-surgery along an annulus, so one component of $\hat{P}^{\prime}$ is essential. It follows that the corresponding component of $P^{\prime}$ is a presurface with lower complexity.

THEOREM 3.4. Suppose $M$ is a hyperbolic 3-manifold. Let $S$ be the set of basic degenerating curves on a genus $g>1$ boundary component $F$ of $M$. Then $|S| \leq k_{g}$ where $k_{g}$ is a constant depending only on $g$.

PROOF. For each $\alpha$ in $S$, fix an essential surface $P_{\alpha}$ as in Lemma 3.3. Let $\partial_{1} \ldots, \partial_{s}$ be those components of $\partial P_{\alpha}$ that are parallel to $\alpha$, and let $\partial_{1}^{\prime}, \ldots, \partial_{t}^{\prime}$ be those that are coplanar with $\alpha$ but not parallel to $\alpha$. Since each $\partial_{i}^{\prime}$ bounds a punctured torus containing $\alpha$, and since these $\partial_{i}^{\prime}$ are mutually disjoint, it is easy to see that they must be parallel to each other. Use $\alpha^{\prime}$ to denote a curve parallel to $\partial_{i}^{\prime}$. Define

$$
\tilde{\alpha}= \begin{cases}\alpha & \text { if } s \geq t \\ \alpha^{\prime} & \text { if } s<t .\end{cases}
$$

Call $\tilde{\alpha}$ the major boundary slope of $P_{\alpha}$. 
LEMMA 3.5. If $\alpha_{1}, \ldots, \alpha_{k}$ are elements in $S$ so that $\tilde{\alpha}_{i}$ is isotopic to $\tilde{\alpha}_{j}$ for all $i, j$ then $k \leq 2 C_{1}$.

PROOF. By isotopies, we may assume without loss of generality that $\tilde{\alpha}_{i}=\tilde{\alpha}_{j}$ for all $i, j$. Write $\tilde{\alpha}=\tilde{\alpha}_{j}$. If $\tilde{\alpha}$ is nonseparating, then by the definition of $\tilde{\alpha}_{j}$ we must have $\tilde{\alpha}=\tilde{\alpha}_{j}=\alpha_{j}$ for all $j$. (Note that $\alpha^{\prime}$ in the definition of $\tilde{\alpha}$ is separating.) If $\tilde{\alpha}$ is separating and also degenerating, then since the $\alpha_{j}$ are basic degenerating curves and are coplanar with $\tilde{\alpha}$, we also have $\tilde{\alpha}=\alpha_{j}$ for all $j$. In these cases, $k=1$.

Now assume $\tilde{\alpha}$ is a separating non-degenerating curve. Then $\alpha_{i}$ must be a non-separating curve in a punctured torus bounded by $\tilde{\alpha}$. By Lemma 1.1, we have $M\left[\alpha_{i}\right]=M[\tilde{\alpha}]\left[\alpha_{i}\right]$, so $\alpha_{i}$ is a degenerating curve on a torus boundary component of the hyperbolic manifold $M[\tilde{\alpha}]$. Since $\dot{\alpha}$ bounds at most two punctured tori, these $\alpha_{i}$ lie in at most two tori. Therefore, by Proposition 2.4, we have $k \leq 2 C_{1}$.

Let $\mathcal{S}^{\prime}=\left\{\tilde{\alpha_{i}}\right\}$ be the set of different major boundary slopes on $F$. By Lemma 3.5 we have

$$
|\mathcal{S}| \leq 2 C_{1}\left|\mathcal{S}^{\prime}\right|
$$

Consider two elements $\tilde{\alpha_{1}}, \tilde{\alpha_{2}} \in \mathcal{S}^{\prime}$. Let $P_{i}=P_{\alpha_{i}}$ be the corresponding essential surfaces. Let $s_{i}$ be the number of boundary components of $P_{i}$ which are parallel to $\tilde{\alpha}_{i}$. Since $\tilde{\alpha}_{i}$ is the major boundary slope, the number of boundary components of $P_{i}$ which are coplanar with $\tilde{\alpha}_{i}$ is at most $2 s_{i}$. Since $P_{i}$ has at most two components not coplanar with $\tilde{\alpha}_{i}$, we have

$$
\left|\partial P_{i}\right| / s_{i} \leq\left(2+2 s_{i}\right) / s_{i} \leq 4 .
$$

Now by Lemma 2.2 we have

$$
\triangle\left(\tilde{\alpha_{1}}, \tilde{\alpha_{2}}\right) \leq 18\left|\partial P_{1}\right|\left|\partial P_{2}\right| / s_{1} s_{2} \leq 288 .
$$

Applying Corollary 3.2 to $S^{\prime}$, we get $\left|\mathcal{S}^{\prime}\right| \leq C(g, 288)$. Therefore, $|\mathcal{S}| \leq$ $2 C_{1} C(g, 288)$. The right hand side depends only on the genus of $F$. This completes the proof of the theorem.

\section{Reducing or $\partial$-reducing handle additions}

Somehow the study of the handle addition problems is inspired by results about Dehn fillings. There are some known results about what happens if 
two Dehn fillings degenerate the manifold in certain senses; see for example $[3,4,8,10]$. It is an interesting problem whether similar results hold for handle additions. The following result is a special case of [8, Theorem 6.1].

THEOREM 4.1. Suppose $X$ is a $\partial$-reducible manifold. Let $K$ be a knot in $X$ such that $X-K$ is irreducible and $\partial$-irreducible. Then a Dehn surgery on $K$ produces a reducible manifold if and only if $K$ is a cable knot, and the surgery slope is that of the cabling annulus.

Let $M=X-\eta(K)$. Then a Dehn surgery on $K$ is the same as a Dehn filling of $M$ along $\partial N(K)$. The filling along a meridian of $K$ produces a $\partial$ reducible manifold and the filling along the cabling slope yields a reducible one. In particular, the geometric intersection number of the two degenerating slopes is one.

We are interested in the problem of what could happen if two degenerating handle additions produce reducible and $\partial$-reducible manifolds respectively. One way to obtain manifolds allowing such handle additions is to drill holes in the above manifolds; see Example 1.2 for details. In that example, the two degenerating curves intersect at a single point. Generally, this may not be true. For example, let $W$ be a reducible and $\partial$-reducible manifold; let $K$ be a trivial knot, and let $\gamma$ be an arc from $K$ to $\partial W$ so that $W-\eta(K \cup \gamma)$ is hyperbolic. By the same method as in Examples 1.1 and 1.2, one can show that infinitely many handle additions produce reducible or $\partial$-reducible manifolds. However, in all these examples, if two degenerating curves have nontrivial intersections, then they are contained in a punctured torus. It turns out that this always happens.

THEOREM 4.2. Suppose $M$ is a hyperbolic manifold, $\alpha, \beta$ are essential closed curves on a non-torus boundary component of $M$, such that $M[\alpha]$ is reducible and $M[\beta]$ is $\partial$-reducible. Then either $\Delta(\alpha, \beta)=0$ or there is a once punctured torus $P$ in $\partial M$ containing both $\alpha$ and $\beta$.

PROOF. Let $F$ be the component of $\partial M$ containing $\alpha$ and $\beta$. We can write $M$ as $M^{\prime} \cup F \times I$, where $F \times I$ is a regular neighbourhood of $F$ in $M$, and $M^{\prime}$ is the closure of $M-F \times I$. Let $K$ be the curve $\alpha \times 1$ on the surface $F^{\prime}=F \times 1$. We consider $\mathrm{K}$ as a knot in $M$. Denote by $X$ the manifold obtained from $M$ by deleting a regular neighbourhood of $K$, then attaching a 2-handle along the curve $\beta$ on $F$. In our earlier notation, $X=(M-\eta(K))[\beta]$.

Denote by $S$ the surface $F^{\prime} \cap X=F^{\prime}-\eta(K)$. It divides $X$ into two parts, $X_{1}$ and $X_{2}$, where $X_{1}=M^{\prime}-\eta(K)$ is homeomorphic to both $M^{\prime}$ and $M$, and 
$X_{2}=(F \times I)[\beta]-\eta(K)$ is homeomorphic to $(F \times I)[\beta]$. Let $m$ be a meridian curve of $K$ on $\partial N(K)$, and let $l$ be a longitude isotopic to the boundaries of $S$. Clearly, the Dehn filled manifold $X[m]$ is exactly equal to $M[\beta]$, so by our assumption it is $\partial$-reducible.

Now consider $X[l]$. The surface $S$ has two boundary components on the torus $\partial N(K)$. They bound disjoint disks $D_{1}$ and $D_{2}$ in the attached solid torus, dividing the solid torus into two 2-handles. Thus, instead of attaching the solid torus, we can attach the 2-handles to $X_{1}$ and $X_{2}$ respectively, then glue the manifolds along the surface $\hat{S}=S \cup D_{1} \cup D_{2}$. Since $\left(X_{1}, l\right) \cong\left(M^{\prime}, K\right) \cong(M, \alpha)$ and $\left(X_{2}, l\right) \cong((F \times I)[\beta], K)$, the two pieces of $X[l]$ cutting along $\hat{S}$ are homeomorphic to $M[\alpha]$ and $(F \times I)[\beta][K]$, respectively. By assumption, $M[\alpha]$ is reducible. Since $(F \times I)[\beta][K]$ has two boundary components, gluing it to a reducible manifold along one of its boundary components will never produce an irreducible manifold. Hence $X[l]$ is reducible.

In summary, we have two Dehn fillings of $X$ along a torus boundary component $\partial N(K)$, one produces a $\partial$-reducible manifold $X[\mathrm{~m}]$, the other produces a reducible manifold $X[l]$. Therefore we can use Theorem 4.1 to conclude that either

(1) $X$ is reducible or $\partial$-reducible, or

(2) $X$ contains an essential annulus $A$ with both boundary components on $\partial N(K)$ and parallel to $l$.

Consider the manifold $X_{2} \cong(F \times I)[\beta]$. The compressing disks of $\partial X_{2}$ are well understood: an essential curve $\gamma$ on $F \times I$ bounds a disk in $X_{2}$ if and only if either (i) $\gamma$ is parallel to $\beta \times 1$, or (ii) $\gamma$ bounds a once-punctured torus $T$ on $F \times 1$ which contains $\beta \times 1$ as a non-separating curve.

We first assume $S=F \times I-\eta(K)$ is compressible in $X_{2}$, and let $\gamma$ be the boundary of a compressing disk. In Case (i), $\gamma$ is parallel to $\beta \times 1$. Since $\gamma$ is disjoint from $K=\alpha \times 1$, we see that $\alpha$ and $\beta$ are disjoint. In Case (ii), if $K$ is not in $T$, then $\alpha$ is disjoint from $\beta$, while if $K$ is in $T$, both $\alpha$ and $\beta$ are in a once-punctured torus. In all cases, the conclusion of the theorem follows.

We now assume $S$ is incompressible in $X_{2}$. Notice that since $\alpha$ is essential and $\partial M$ is incompressible, $\partial M-\alpha$ is incompressible in $M$, so $S$ is also incompressible in $X_{1}$. By assumption, $X_{1} \cong M$ is irreducible. As $X_{2}$ is obtained from $F \times I$ by attaching a single 2-handle, it is easy to see that $X_{2}$ is also irreducible. Therefore by a standard innermost circle argument one can show that $X=X_{1} \cup X_{2}$ is irreducible and boundary irreducible. This completes the proof of Case (1) above. 
Now assume Case (2) and let $A$ be an annulus such that $\partial A$ lies on $\partial N(K)$ and is disjoint from $S$. Since $S$ is incompressible, and $X_{1}, X_{2}$ are irreducible, by an isotopy we may assume $A \cap S$ consists of essential circles in $A$, so all components of $A \cap X_{i}$ are annuli. We may assume the $A$ has been isotoped so that $A \cap S$ is minimal. Since $X_{1} \cong M$ is hyperbolic, and $X_{2}=(F \times I)[\beta]$ is a compression body, neither of them contains essential annuli. In particular $A \cap S \neq \emptyset$. Let $A^{\prime}$ be a component of $A \cap X_{i}$ which has one boundary component on $\partial A$. Since $A^{\prime}$ is incompressible (otherwise $S$ would be compressible), it is $\partial$-compressible. But a $\partial$-compression would yield an embedded disk with boundary on $S$. Since $S$ is incompressible and the $X_{i}$ are irreducible, the disk is parallel to a disk in $S$. Therefore, $A^{\prime}$ is $\partial$-parallel. By an isotopy of $A$, we can reduce the number of components in $A \cap S$, contradicting the choice of $A$.

The second conclusion of the theorem can be further clarified. Actually, from the proof we have the following corollary, which shows that Example 1.2 and the construction given prior to Theorem 4.2 have produced all such manifolds.

COROLlaRY 4.3. Let $M, \alpha, \beta$ be as in the theorem. If $\alpha, \beta$ are contained in $a$ once-punctured torus $P$, then either $M[\partial P]$ is reducible or $\partial$-reducible, or $M[\partial P]$ contains a cable space. Hence, if $\Delta(\alpha, \beta)>1$ then $M[\partial P]$ must be either reducible or $\partial$-reducible.

COROLLARY 4.4. Let $M, \alpha, \beta$ be as in the theorem. If either $\alpha$ or $\beta$ is a basic degenerating curve, then $\Delta(\alpha, \beta)=0$.

In particular, if one of $\alpha$ and $\beta$ is a separating curve then $\Delta(\alpha, \beta)=0$.

Proof. By Corollary 4.3 , if $\Delta(\alpha, \beta) \neq 0$ then $M[\partial P]$ is reducible, $\partial-$ reducible, or contains a cable space. In all cases, $M[\partial P]$ is non-hyperbolic.

We suspect that a result similar to Theorem 4.2 might be true for all degenerating handle additions.

CONJECTURE 1. If $\alpha$ and $\beta$ are both degenerating curves, then either $\Delta(\alpha, \beta)=0$ or they can be isotoped into a once-punctured torus.

Conjecture 1 seems still far from being solved. A consequence of it would be the following. If $\alpha$ and $\beta$ are both in a punctured torus $P$, then $M[\alpha]=$ $M[\partial P][\alpha]$, and $M[\beta]=M[\partial P][\beta]$ by Lemma 1 . If $M[\partial P]$ is not hyperbolic, then $\partial P$ is a separating degenerating curve, so neither $\alpha$ nor $\beta$ is basic. If $M[\partial P]$ is hyperbolic, then by Gordon's Theorem [3, Theorem 3.4, 3.5], $\Delta(\alpha, \beta) \leq 5$. So Conjecture 1 implies the following: 
CONJECTURE 2. If $\alpha$ and $\beta$ are basic degenerating curves on $\partial M$, then $\triangle(\alpha, \beta) \leq 5$.

If the conjecture is false, it would still be interesting to know if a universal upper bound for $\Delta(\alpha, \beta)$ exists, and to determine the least upper bound. By a similar method as in the proof of Theorem 3.4, we can show that if $\alpha, \beta$ are separating degenerating curves, then $\Delta(\alpha, \beta) \leq 14$. As this is not expected to be the best possible, we omit the proof.

\section{Added in proof}

A recent example of Mario Eudave-Munoz provides a counterexample to Conjecture 1. But it would still be interesting to know whether it is true when both of the resulting manifolds are reducible or boundary reducible. Conjecture 2 remains open.

\section{References}

[1] S. Bleiler and C. Hodgson, 'Spherical space forms and Dehn filling', preprint.

[2] C. Gordon, 'Boundary slopes of punctured tori in 3-manifolds', preprint.

[3] _ 'Dehn surgery on knots', in: Proceedings of the International Congress of Mathematicians, Kyoto 1990 (Springer, Berlin, 1991) pp. 631-642.

[4] C. Gordon and J. Luecke, 'Reducible manifolds and Dehn surgery', preprint.

[5] J. Hempel, 3-manifolds, Ann. of Math. Studies 86 (Princeton Univ. Press, Princeton, 1976).

[6] W. Jaco, 'Lectures on three-manifold topology', Regional Conference Series in Mathematics 43 (1981).

[7] R. Myers, 'Excellent 1-manifolds in compact 3-manifolds', Top. Appl., to appear.

[8] M. Scharlemann, 'Producing reducible 3-manifolds by surgery on a knot', Topology 29 (1990), 481-500.

[9] W. Thurston, The geometry and topology of 3-manifolds (Lecture notes, Princeton University, 1978).

[10] Y-Q. Wu, 'Incompressibility of surfaces in surgered 3-manifolds', Topology 31 (1992), 271-280.

Department of Mathematics

University of California

Santa Barbara, CA 93106
Department of Mathematics

University of California

Santa Barbara, CA 93106 